\title{
Trends of breast cancer in premenopausal women over two decades
}

\author{
Ana Sofia Pais ${ }^{1,2 *}$, Simone Subtil ${ }^{1,2}$ and Margarida Figueiredo-Dias ${ }^{1,2}$ \\ ${ }^{1}$ Gynecology Department, Centro Hospitalar e Universitário de Coimbra, Coimbra, Portugal \\ ${ }^{2}$ Faculty of Medicine, University of Coimbra, FMUC, Portugal
}

\begin{abstract}
Objective: Knowledge of breast cancer patterns over time allows a better understanding of the disease, with benefits for research and treatment improvement. This study analyses the evolution of epidemiological and pathological patterns in premenopausal breast cancer over two decades.

Material and methods: Medical records from premenopausal breast cancer patients in our department were analysed. Two comparison groups over time were established: group A (1995-1998) and B (2008-2011).

Results: Group A included 105 patients and group B 282, revealing an increasing incidence. A significant increase in patient age at diagnosis, age at first pregnancy, breastfeeding and hormonal contraception lifespan were observed, as well as a significant decrease in age of menarche. Imagiological diagnosis increased over time, resulting in an earlier stage disease. Surgical treatment has become predominantly conservative and hormone therapy has increased markedly. Overall prognosis was improved, followed by a significant decrease in recurrence and mortality rates.
\end{abstract}

Conclusion: From 1995 to 2011, significant changes are reported in premenopausal breast cancer in women, which reflects the enormous and significant improvement in healthcare.

\section{Introduction}

According to data from Globocan 2012, an estimated 1.67 million new breast cancers were diagnosed during 2012 [1]. It is the most common cancer in women both in developed regions and in countries with emerging economies, with a slight majority of cases occurring in wealthy nations [2]. The rate of breast cancer is increasing globally, with a rising incidence in patients younger than 50 years of age, regarding the SEER (Surveillance Epidemiology and End Results) Program [3].

Breast cancer is currently considered a highly heterogeneous disease, classified by distinct molecular profiles and requiring individualized treatment. Differences in its incidence, pathology, and mortality have been noticed in terms of different regions and ethnicity, which may be related to genetic background, socioeconomic development and lifestyle patterns [4,5]. However, concerning advanced-stage breast cancer, the risk seems to be independent of intrinsic or extrinsic patterns, emphasizing the demand to improve screening practices and clinical treatment [6]. According to the 2015 Guideline Update from the American Cancer Society, breast cancer screening in women with an average risk should start aged 45 years. However, women from 40 to 44 years old should have the opportunity to begin annual screening. At ages $45-54$ it is recommended an annual mammography. Women 55 years and older should transition to biennial screening or have the opportunity to continue screening annually [7]. In our country, the breast cancer screening programme started in 1990 and recommends biennial mammography from 50 to 69 years, with a starting age at 45 years in the central region of the country.

Although the risk of breast cancer increases with age, approximately $35 \%$ of breast cancers occur during the reproductive and perimenopausal years [3]. Breast cancer in young women is worthy of special attention as it often represents a distinct entity, due to its unique and complex issues, namely risk factors, clinical outcomes, and tumour biology $[8,9]$. Premenopausal patients should be considered a particular group, as the tumour has frequently reached a more advanced stage and carcinomas seem to have more aggressive behaviour $[8,10]$.

In this particular population, knowledge the evolution of breast cancer patterns over time allows a better understanding of the disease, with benefits for research and treatment improvement.

\section{Objectives}

This study reviews trends premenopausal breast cancer epidemiological and clinicopathological patterns over two decades.

\section{Materials and methods}

\section{Study subjects}

A retrospective study was performed based on data collected through medical records from a university hospital in the central region of a Southern European country. The inclusion criteria included breast

*Correspondence to: Ana Sofia Pais, Gynecology Department, Centro Hospitalar e Universitário de Coimbra, Praceta Prof. Mota Pinto, 3000-075 Coimbra, Portugal, E-mail: asfpais@uc.pt

Key words: breast cancer, epidemiology, clinicopathological parameters, premenopausal women

Received: March 13, 2019; Accepted: April 11, 2019; Published: April 15, 2019 
cancer diagnosis from 1995 to 2011, female and premenopausal status. Two groups were established: group A, consisting of breast cancers diagnosed from 1995 to 1998, and group B, from 2008 to 2011.

\section{Study design}

This cross-sectional study employed a retrospective chart review approach and a comparative analysis of the groups.

\section{Data collection}

A customized retrospective review was used to collect information from patient records. The principal investigator and medical doctors from the department were responsible for the data collection strategy, the type of variables, the accuracy required, the collection point and the skill of the enumerator. The information included demographic characteristics-age, ethnicity and body mass index; gynecologic and obstetric data-age of menarche, length of use of hormonal contraception, parity, age at first pregnancy and breastfeeding; clinical characteristics of the tumour-palpable/non-palpable and stage (TNM); histopathological features-tumour type, tumour grade, lymph node status, oestrogen and progesterone receptors (ER and PR), and HER2 receptors status; therapeutic protocols; mortality and recurrence rate.

\section{Statistical analysis}

Means and standard deviations (SD) were described for the continuous variables with normal distribution. An independent t-test was used to determine the difference between respective groups. Frequencies and proportions were used for categorical variables. The differences were determined by a chi-squared test or a Fisher exact test. Differences in overall survival and relapse-free time between groups were investigated using Kaplan-Meier curves. A p value of $<0.05$ was considered to be statistically significant. All analyses were performed using SPSS software, version 22.0.

\section{Ethical considerations}

The study was performed in accordance with the Helsinki Declaration and with approval of the Institutional Review Board. Since the study involved completely de-identified data extraction from electronic medical records, patient consent was not required.

\section{Results}

The study included 387 women with premenopausal breast cancer, 105 diagnosed from 1995 to 1998 (group A) and 282 from 2008 to 2011 (group B), revealing an increased incidence during the time lapse.

The age at diagnosis was $42.6 \pm 5.9$ years in group $\mathrm{A}$ and $45.3 \pm 6.2$ years in group $\mathrm{B}(\mathrm{p}<0.001)$ (Table 1$)$. Regarding clinical data, a significant decrease in age of menarche (12.9 \pm 1.6 vs $12.4 \pm 1.4$ years, $\mathrm{p}=0.014$ ) was observed over time. Hormonal contraception users were similar during the period of the study; however, length of use of birth control pills significantly increased from group A to group B, with an average duration of $7.3 \pm 5.7$ to $10.9 \pm 5.1$ years, $p<0.001$. Assessing obstetric data, the number of deliveries was higher in the most recent group ( $\mathrm{p}<0.001$ ), as well as the average age of first-time mothers: 23.6 years in 1995-1998, compared with 24.9 years in 2008-2011 ( $\mathrm{p}=0.014)$. Breastfeeding was less frequent previously ( $49 \%$ vs $63 \%, \mathrm{p}=0.015$ ), but a similar duration was registered during time of the study period (Table $1)$.

Concerning tumour characteristics, some differences were observed. Palpable tumours tended to decrease over the study period
Table 1. Epidemiological, clinical and pathological patterns overtime, between 1995-1998 and 2008-2011

\begin{tabular}{|c|c|c|c|}
\hline Factors & $\begin{array}{c}A \\
(1995-1998) \\
n=105\end{array}$ & $\begin{array}{c}\text { B } \\
(2008-2011) \\
n=282\end{array}$ & $\mathbf{p}$ \\
\hline Age (Mean, SD) & $42.6 \pm 5.9$ & $45.3 \pm 6.2$ & $<0.001$ \\
\hline \multicolumn{4}{|l|}{ Gynaecological data } \\
\hline Age of Menarche (Mean, SD) & $12.9 \pm 1.6$ & $12.4 \pm 1.4$ & 0.014 \\
\hline Hormonal contraception use & $53 \%(\mathrm{n}=56)$ & $50 \%(\mathrm{n}=142)$ & n.s. \\
\hline $\begin{array}{l}\text { Length of use of birth } \\
\text { Control pills (Mean, SD) }\end{array}$ & $7.3 \pm 5.7$ & $10.9 \pm 5.1$ & $<0.001$ \\
\hline \multicolumn{4}{|l|}{ Obstetric data } \\
\hline Parity & - & - & $<0.001$ \\
\hline 0 & $22 \%(\mathrm{n}=21)$ & $13 \%(n=32)$ & - \\
\hline 1 & $77 \%(n=74)$ & $51 \%(n=129)$ & - \\
\hline 2 & $1 \%(n=1)$ & $36 \%(n=89)$ & - \\
\hline Age at first pregnancy (Mean, SD) & $23.6 \pm 3.1$ & $24.9 \pm 4.9$ & 0.014 \\
\hline Breastfeeding & $49 \%(n=52)$ & $63 \%(n=178)$ & 0.015 \\
\hline Breast feeding duration & $7.2 \pm 5.3$ & $8.6 \pm 7.0$ & n.s. \\
\hline \multicolumn{4}{|l|}{ Tumour characteristics } \\
\hline Palpable tumours & $65 \%(n=68)$ & $61 \%(n=172)$ & n.s. \\
\hline $\begin{array}{l}\text { Imagiological diagnosis of } \\
\text { Subclinical tumors }\end{array}$ & $39 \%(n=41)$ & $89 \%(\mathrm{n}=252)$ & $<0.001$ \\
\hline \multicolumn{3}{|l|}{ Cancer stage } & 0.029 \\
\hline I & $47 \%(n=49)$ & $57 \%(\mathrm{n}=151)$ & - \\
\hline II & $36 \%(n=38)$ & $32 \%(\mathrm{n}=86)$ & - \\
\hline III & $14 \%(n=15)$ & $5 \%(n=12)$ & - \\
\hline IV & $4 \%(n=4)$ & $6 \%(n=18)$ & - \\
\hline \multicolumn{4}{|l|}{ Histopathological analysis } \\
\hline In situ carcinoma association & $30 \%(n=32)$ & $20 \%(\mathrm{n}=57)$ & n.s. \\
\hline Differentiation grade & - & - & n.s. \\
\hline Well differentiated (G1) & $25 \%(n=26)$ & $26 \%(n=60)$ & - \\
\hline Moderately differentiated (G2) & $49 \%(n=47)$ & $48 \%(n=110)$ & - \\
\hline Poorly differentiated (G3) & $23 \%(\mathrm{n}=22)$ & $26 \%(\mathrm{n}=59)$ & - \\
\hline \multicolumn{4}{|l|}{ Molecular subtypes } \\
\hline Luminal A & - & $62 \%(n=155)$ & - \\
\hline Basal-like & - & $22 \%(\mathrm{n}=56)$ & - \\
\hline Luminal B & - & $11 \%(n=26)$ & - \\
\hline HER 2+ & - & $5 \%(n=13)$ & - \\
\hline Axillary invasion & $42 \%(n=44)$ & $31 \%(n=86)$ & 0.05 \\
\hline
\end{tabular}

as imaging diagnosis of subclinical tumours increased significantly (group A-39\% and group B-89\%, $\mathrm{p}<0.001$ ).

Breast cancer is now diagnosed at an earlier stage: at stage $\mathrm{I}$ in $47 \%$ in group A vs $57 \%$ in group B, stage II in $36 \%$ vs $32 \%$, stage III in $14 \%$ vs $5 \%$ and stage IV in $4 \%$ vs $6 \%$, respectively ( $\mathrm{p}=0.029$ ).

According to pathological analysis of the tumours, invasive ductal carcinoma still remains the most common histological type and in situ carcinoma is associated with $25 \%$ of the cases, with no differences registered between the groups $(\mathrm{p}=\mathrm{n}$.s.). The degree of differentiation did not change during the study period, being well differentiated (G1)$25 \%$ in group A $v s 26 \%$ in group B and poorly differentiated (G3)-23\% vs $26 \%$. No comparative study was performed regarding molecular subtypes, as in the group A time range only oestrogen receptor (ER) expression was available and no routine progesterone receptors (PR) were assessed. From 2008 to 2011, the luminal A tumour was the most frequent (62\%), followed by basal-like (22\%), luminal B (11\%) and HER2+(5\%). Axillary invasion was significantly lower in group B (31\%) when compared to group A $(42 \%),(\mathrm{p}<0.05)$, according to the current earlier detection. 
Considering therapeutic protocols, surgical treatment is predominantly conservative in patients diagnosed from 2008 to 2011 ( $44 \%$ vs $12 \%$ in formerly patients, $\mathrm{p}<0.001$ ). Sentinel node biopsy emerged during the study period, starting during the year 2000 . The same ratio of patients from both groups received neoadjuvant treatment, namely chemotherapy, and a significant improvement in complete pathological response regarding recent data was registered $(\mathrm{p}<0.05)$. The use of adjuvant radiation therapy and chemotherapy was similar over time, but hormone therapy significantly increased during the study period ( $59 \%$ vs $72 \%, \mathrm{p}=0.015)$.

Overall prognosis improved, and a significant decrease in locoregional and distant recurrence (9\% vs $1,4 \%, \mathrm{p}=0.001$ and $27 \%$ vs $14 \%, \mathrm{p}=0.003)$ and increase relapse-free time $(\mathrm{p}<0.001)$ were observed (Table 2). Mortality rate significantly decreased comparing earlier years $(28 \%)$ to recent data $(9 \%),(\mathrm{p}<0.001)$; however, a similar free-survival time was documented with Kaplan-Meier analysis (Figures 1 and 2).

\section{Discussion}

Epidemiological and clinicopathological trends of premenopausal breast cancer during two decades deserve special attention. According to worldwide statistics, a considerable increase in the breast cancer incidence rate was observed in the study population from 1995 to 2001 $[1,3]$. During this time, the diagnosis of breast cancer in premenopausal patients doubled at the University Hospital Centre of Coimbra, in the central region of a Southern European country. Worldwide, the rising incidence is also found in patients younger than 50 years old [3].

In the study population, the mean age at diagnosis of premenopausal breast cancer increased 2.5 years. A similar study, comparing the period from 1974-1978 to 1999-2003, observed that the average age at diagnosis increased by 2 years [11]. From 1998 until 2008, the mean age at diagnosis remains 61 years, according to American epidemiological

Table 2. Overall prognosis

\begin{tabular}{|c|c|c|c|}
\hline Overall prognosis & $\begin{array}{c}\text { A } \\
(\mathbf{1 9 9 5 - 1 9 9 8 )} \\
\mathbf{n = 1 0 5}\end{array}$ & $\begin{array}{c}\mathbf{B} \\
\mathbf{( 2 0 0 8 - 2 0 1 1}) \\
\mathbf{n}=\mathbf{2 8 2}\end{array}$ & $\mathbf{p}$ \\
\hline Loco-regional recurrence & $9 \%(\mathrm{n}=9)$ & $1.4 \%(\mathrm{n}=4)$ & 0.001 \\
\hline Distant recurrence & $27 \%(\mathrm{n}=28)$ & $14 \%(\mathrm{n}=39)$ & 0.003 \\
\hline Mortality rate & $28 \%(\mathrm{n}=29)$ & $9 \%(\mathrm{n}=25)$ & $<0.001$ \\
\hline
\end{tabular}

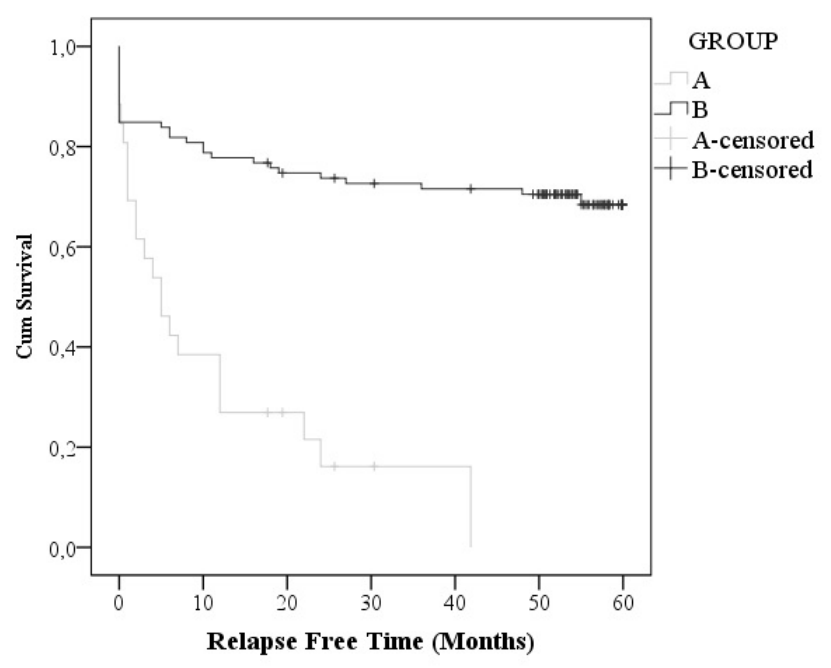

Figure 1. Relapse-free time, Kaplan-Meier curve

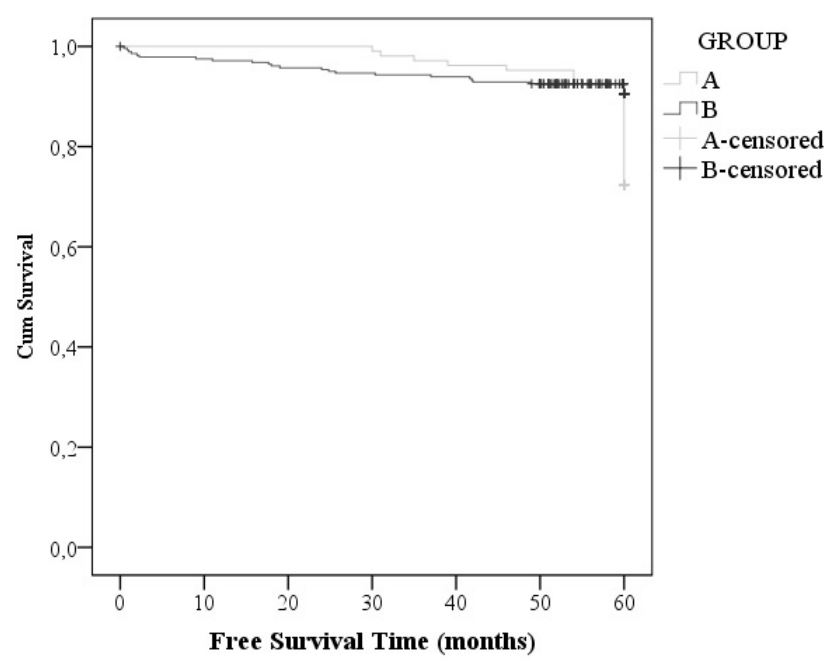

Figure 2. Free survival time, Kaplan-Meier curve

data [3]. In this study, it can be explained by the national screening program, which starts at 45 years of age. Breast cancer risk factors can be divided into un-modifiable and modifiable risk factors. Ethnicity, gender, age, inherited genes, family history, personal history of breast cancer or certain benign breast conditions, dense breast tissue, early menarche and breast radiation at young age usually are considered un-modifiable risk factors. On the other hand, risk factors related to lifestyle are linked to nulliparity or delayed pregnancy, hormonal therapy, absence of breastfeeding, smoking, alcohol, being overweight or obese and lack of exercise. Regarding menopausal status, risk factors are similar, except where nulliparity is concerned [12,13]. Menstrual and reproductive conditions depict the most significant hormonal risk factors in premenopausal breast cancer women. Younger age at menarche, nulliparity, older age at first live birth and no breastfeeding have been consistently related to increased breast cancer risk. In this retrospective study, there was a decrease in age of menarche and an increase in age at first pregnancy. Postponing motherhood is observed worldwide [14], but it is a modifiable risk factor for breast cancer. In contrast, breastfeeding, although considered a protective breast cancer feature, increased during the time of the study. Breast cancer risk is modestly elevated in women who are currently using combined oral contraceptives or have used them during the past 10 years [15]. The present cross-sectional study emphasizes that the rate of contraceptive pills use was similar, but the time range of use was higher in recent data, possibly related to the increased age at first pregnancy.

Over two decades, an improvement in diagnosis was observed regarding early detection, reflecting real advances in the Portuguese health system organization. A breast cancer screening programme was implemented in the central region of Portugal in the nineties; however, it only includes women older than 45 years of age. Thus, breast cancer is diagnosed at an early stage in the most recent study population, reflecting an improvement of imagiological diagnosis and resulting in a decrease in palpable tumours and axillary invasion. Regarding metastatic breast cancer, a slight increase was observed during the study period, which is consistent with the literature, possibly due to aggressive tumour characteristics in younger women $[6,8,10]$. Broadening the screening programme and starting screening mammography at age 40, as suggested by American Cancer Society, would be a valuable option. Histopathological tumour characteristics remain similar, regarding 
histological type, in situ carcinoma association and differentiation grade [16]. Further studies are needed to analyse the evolution pattern of molecular subtypes. Treatment protocols have been modified over the last twenty years along with updated guidelines due to advances in knowledge of diseases and new medicines [17]. A significant difference in surgical procedures was observed over time regarding conservative surgery, as performed worldwide [18]. Selection for neoadjuvant and adjuvant treatment remained constant, except for hormone therapy, which increased in premenopausal breast cancer patients over time.

In spite of early stage breast cancer diagnosis, neoadjuvant chemotherapy is similar, in order to permit tumour downstaging, allowing conservative surgery and better cosmetic results [19].

Despite the rising incidence of premenopausal breast cancer, modification of diagnosis and treatment protocols has allowed an overall improvement in prognosis. Recurrence is less frequent and the mortality rate lower, yet with a similar free survival time, as observed for cancer overall $[1,3,13]$.

\section{Conclusion}

In conclusion, from 1995 to 2011, significant changes are reported in breast cancer in premenopausal women, such as increased incidence, earlier stage diagnosis, predominantly conservative surgical treatment and overall improvement in prognosis. It reflects the enormous effort and significant improvement in healthcare concerning prevention, diagnosis and treatment in Southern European countries as well as in the Portugal health system, particularly concerning the central region of the country. An enlarged study, based on a wide-ranging breast cancer registry in Portugal, is ongoing, comprising data from the whole country and the Portuguese islands.

\section{Financial disclosure}

The authors declare no financial support for the publication, participation of research institutions, associations and other parties.

\section{References}

1. Breast cancer: Estimated incidence, mortality and prevalence worldwide in 2012.

2. Ferlay J, Soerjomataram I, Dikshit R, Eser S, Mathers C, et al. (2015) Cancer incidence and mortality worldwide: Sources, methods and major patterns in GLOBOCAN 2012. Int J Cancer 386: E359-E386. [Crossref]

3. Cancer of the female breast (Invasive): Age-adjusted U.S. death rates by year, race and age.
4. Si W, LiY, Han Y, Zhang F, Wang Y, et al. (2015) Epidemiological and clinicopathological trends of breast cancer in chinese patients during 1993 to 2013 - A retrospective study. Medicine (Baltimore) 94: 1-7.

5. Bhikoo R, Srinivasa S, Yu TC, Moss D, Hill AG (2011) Systematic review of breast cancer biology in developing countries (Part 2): Asian subcontinent and south east Asia. Cancers (Basel) 3: 2382-2401.

6. Merkin SS, Stevenson L, Powe N (2002) Geographic socioeconomic status, race, and advanced-stage breast cancer in New York city. Res Pract 92: 64-70.

7. Oeffinger KC, Fontham ETH, Etzioni R, Herzig A, Michaelson JS, et al. (2015) Breast cancer screening for women at average risk 2015 guideline update from the American cancer society. Clin Rev Educ 314: 1599-1614. [Crossref]

8. Gabriel CA, Domchek SM (2010) Breast cancer in young women. Breast Cancer Res 12: $1-10$

9. Dartois L, Fagherazzi G, Baglietto L, Boutron-Ruault MC, Delaloge S, et al. (2016) Proportion of premenopausal and postmenopausal breast cancers attributable to known risk factors: Estimates from the E3N-EPIC cohort. Int J Cancer 138: 2415-2427. [Crossref]

10. Banz-Jansen C, Heinrichs A, Hedderich M, Waldmann A, Dittmer C, et al. (2012) Characteristics and therapy of premenopausal patients with early-onset breast cancer in Germany. Arch Gynecol Obs 286: 489-493. [Crossref]

11. Hayat MJ, Howlader N, Reichman ME, Edwards BK (2007) Cancer statistics, trends, and multiple primary cancer analyses from the surveillance, epidemiology, and end results (SEER) program. Oncologist 2007: 20-37.

12. Butt Z, Haider SF, Arif S, Khan MR, Ashfaq U, et al. (2012) Breast cancer risk factors: a comparison between pre-menopausal and post-menopausal women. J Pak Med Assoc 62: 120-124. [Crossref]

13. Taira N, Arai M, Ikeda M, Iwasaki M (2016) The Japanese breast cancer society clinical practice guidelines for epidemiology and prevention of breast cancer, 2015 edition. Breast Cancer.

14. Mathews TJ, Hamilton BE (2016) Mean age of mothers is on the rise: United States, 2000-2014. NCHS Data Brief 2016: 1-8. [Crossref]

15. Collaborative group on hormonal factors in breast cancer (1996) Breast cancer and hormonal contraceptives: collaborative reanalysis of individual data on 53297 women with breast cancer and 100239 women without breast cancer from 54 epidemiological studies. Lancet 347: 1713-1727. [Crossref]

16. Wilailak S, Lertchaipattanakul N (2016) The epidemiologic status of gynecologic cancer in Thailand. J Gynecol Oncol 27: e65. [Crossref]

17. Coates AS, Winer EP, Goldhirsch A, Gelber RD, Gnant M, et al. (2015) Tailoring therapies - improving the management of early breast cancer: St Gallen international expert consensus on the primary therapy of early breast cancer 2015. Ann Oncol 26: 1533-1546. [Crossref]

18. Karasawa K, Obara T, Shimizu T, Haga S, Okamoto T, et al. (2003) Outcome of breastconserving therapy in the Tokyo Women's medical university breast cancer society experience. Breast Cancer 10: 341-348.

19. Criscitiello C, Curigliano G, Burstein HJ, Wong S (2016) Breast conservation following neoadjuvant therapy for breast cancer in the modern era: Are we losing the opportunity? Eur J Surg Oncol 42: 1780-1786.

Copyright: (C2019 Pais AS. This is an open-access article distributed under the terms of the Creative Commons Attribution License, which permits unrestricted use, distribution, and reproduction in any medium, provided the original author and source are credited. 